\title{
Eddy Currents Based Stray Loss Model For Variable Load Conditions of Medium Power Induction Motors
}

\author{
Rajendra Kumar, Praveen Kumar, Senior Member, IEEE, Teruo Kanekawa, and Koji Oishi
}

\begin{abstract}
This paper presents an eddy current based stray loss model for induction motors taking into account the impact of motor's loading. The model uses the various motor inductances and, other nameplate data of an induction motor as primary variables. The Magnetic field distribution in a motor is prominently affected by the stator and rotor slot geometries. Distortions in the magnetic field have a direct impact on SL as well as on various inductances of the motor. The development and validation of the model is accomplished by testing total 26 numbers of induction motors of different geometries and materials at different loading conditions. In a further step, the applicability of the model in designing an IM is presented by re-designing a $75 \mathrm{~kW}$ induction motor and comparing the experimental test results with the initial IM.
\end{abstract}

Index Terms-Induction motors, stray losses, equivalent circuit parameters, core loss, modelling

\section{INTRODUCTION}

D Esign and operation of energy efficient motor-driven systems (MDS) require accurate estimates of all the losses incurred in the motor. Mechanical, stator copper, rotor copper, iron, and stray load losses (SL) are the five significant losses noted for the motors [1]. All these losses, except the SL, are thoroughly analyzed and reported in the literature. Several fairly accurate models exist for these losses, whereas the formulation of SL is still a challenge [2]. This is due to multiple factors influencing the SL. These factors include slot MMF and permeance harmonics due to stator and rotor slots, magnetic non-linearity of the ferromagnetic core, and various leakage fluxes [3], [4]. The typical value of the SL in a motor, at its rated operation, lies between $0.5 \%$ and $2.5 \%$ of the motor's power rating [5], [6], significant enough to affect the motor's efficiency estimation. Further, the SL becomes more critical for premium efficiency motors as all the other losses are small.

Literature broadly discusses SL's evaluation by four approaches: viz. testings [7], numerical [8], equivalent circuit representation [9], and empirical formulation [10], [11] . Among these methods, testing is not applicable in design phase. High implementation time, large data storage, and complex computation of numerical methods led to empirical representation of SL. Given the fact that, in industrial applications, $44 \%$ of machines in the industrial sector run with loads less than or equal to $40 \%$ of their rated value [12]. the available literature, in our findings, still lacks an SL model, which can:
1) approximate the $S L$ values for different load-conditions of an IM while designing it so that SL can be minimised in the design phase by revamping the IM's geometry for a wide range of its operation.

2) distinguish the IMs of the same power rating with respect to (w.r.t.) their SL values. For example, two motors of the same power ratings, number of slots, and material but different slot shapes would show different SL values. The SL model should apprehend this.

3) also be used for motors already in operation, whose detailed design and material data are not available except for the name-plate data;

This paper presents an ECP based SL model to cater to all the above requirements mentioned with the accomplishment of the following objectives:

1) to assess and formulate the SL variation with motors' loading.

2) to assist the motor designer to evaluate and minimise the $S L$ while designing the motor using the formulated $S L$ model. The model should demonstrate a good sensitivity with the motor's geometry to lead the designer to a minimal SL design.

3) propose an SL model applicable to a wide range of medium power motors i.e., from $0.75 \mathrm{~kW}$ to $375 \mathrm{~kW}$.

ECPs and nameplate details are chosen as the primary variables for the proposed SL model. The ECPs are the function of motor's geometry and, can be evaluated at both the stages i.e. during the design(from the geometry details of the motor [9], [13]) and, after the prototyping (by standard tests [5], [14]).

The paper's organisation is as follows: Section II describes SL variation with the motor's loading. Section III pertains to the model formulation based upon the experimental results of section II. Validation of the proposed formulation is presented in Section IV with using a set of eight IMs of different slot geometries and rotor materials and, by re-designing a $75 \mathrm{~kW}$ IM. Finally, section V concludes the paper.

\section{Measurement of SL}

To trace and formate the SL in an IM and its variation with loading, two sets (i.e., set-1 and set-2) of measured SL values of a total of 26 IMs are used. Set-1 comprises rated SL values of 18 IMs of different geometries, power ratings and number of poles. Set-2, used to study the SL variation with 
TABLE I

IMs’ Test SAMPLE Distribution For RATED SL

\begin{tabular}{cccc}
\hline \hline Poles & $\begin{array}{c}\text { Number } \\
\text { of IMs }\end{array}$ & $\begin{array}{c}\text { Minimum } \\
\text { power }\end{array}$ & $\begin{array}{c}\text { Maximum } \\
\text { power }\end{array}$ \\
\hline 2 & 3 & $75 \mathrm{~kW}$ & $160 \mathrm{~kW}$ \\
4 & 10 & $75 \mathrm{~kW}$ & $375 \mathrm{~kW}$ \\
6 & 5 & $75 \mathrm{~kW}$ & $200 \mathrm{~kW}$ \\
\hline \hline
\end{tabular}

loading, belongs to the eight IMs of the same power ratings, the same number of poles, same outer dimensions but different slot shapes. Subsections A and B present the SL measurements for set-1 and set-2, respectively.

\section{A. SL values at rated loadings}

Variation of SL (at rated $P_{\text {Out }}$ ) among the IMs is figured out using the test data of set-1. Sample distribution of the set-1 is given in Table I. Nameplate details of these IMs are given in annexure-1. All these 18 IMs of set- 1 are tested by TMEICMedium rotating machinery department. SL values for these IMs are measured from the load test with torque measurement as per the section 8.2.2.5.1 of IEC60034-2-1 and, they are shown in Fig. 1.

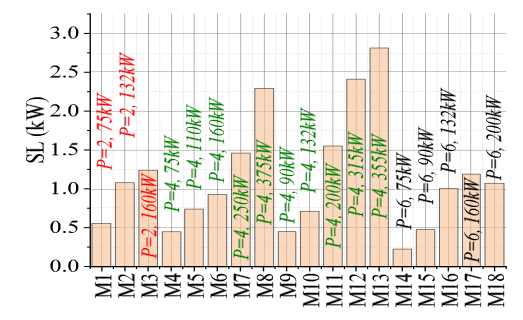

Fig. 1. Test bench with the $75 \mathrm{~kW}$ IM

\section{B. SL variation with loading}

Eight cage-bar induction motors are prototyped to understand the trend of SL variation with the loading of IM. The set of these motors (i.e., set-2) consists:

- eight motors of same power ratings but different slot geometries.

- four motors of same slot geometries (and power ratings) but different rotor material, i.e. two aluminium rotor motors and two copper rotor motors.

The purpose of prototyping eight such diverse IMs is to check the model's sensitivity to SL when motors are very similar except for the slot geometries. Nameplate data of these motors are given in annexure-2. All these $2.5 \mathrm{~kW}$ rating motors have the same diameters, stack length, slot area and end-rings area. The only difference is in the slot geometries and rotor bar material. These motors (Fig. 3(a)) are tested for SL at different operating points (including the rated) as per the relevant and latest test standard-IEC60034-2-1. The test procedure, test results are detailed in the following sub-section.

\section{Experiments and SL test results}

The SLs, for the eight IMs (mentioned in subsection B) are measured using the Eh-star test, following the procedure given

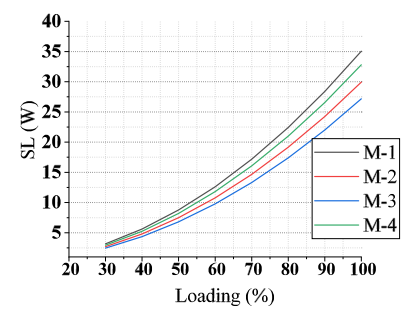

(a)

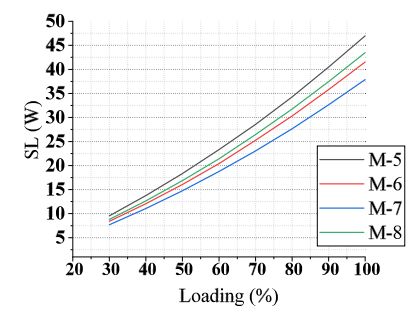

(b)
Fig. 2. Test results:variation of SL with loading for the 2.5kW IMs: (a) $\mathrm{Cu}$ motors, (b) Al motors

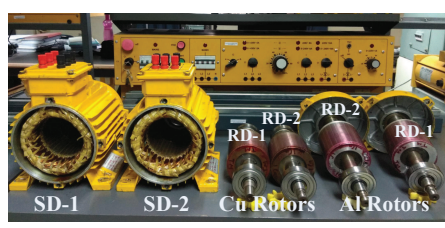

(a)

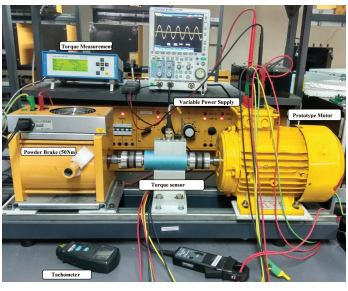

(b)
Fig. 3. (a) $2.5 \mathrm{~kW}, 4 p \mathrm{IMs}$, (b) Test setup

in [5]. The test set-up is shown in Fig. 3(b). The analysis of the results shown in Fig.4(a)-4(b) show that:

- even though the motors M1 to M8 have the same rated output power, the SL is different.

- SL of a motor does not vary in the same proportion of output power (i.e. $\left.\frac{\partial(S L)}{S L} \neq \frac{\partial(\text { Pout })}{\text { Pout }}\right)$, which implies that the relationship between SL and $P_{\text {Out }}$ is not linear. The SL varies as an exponential function of output power, and hence a percentage-based formulation of SL w.r.t. $P_{\text {Out }}$ is not justified for variable load scenarios.

- the motors with aluminium rotors show higher SL values, vis-a-vie copper rotors, for the same output power. This is because the aluminium rotor motors, as opposed to copper rotor motors, run at a relatively higher slip for the same $P_{\text {Out }}$. As a result of this, the stator current and the leakage fluxes increase, resulting in higher SL.

An empirical relation of SL is formulated based on the above observations and rated SL values of set-1. The next section explains the model's development in detail.

\section{Proposed Modeling of SL For VARiable LOADING CONDITIONS}

This section presents the formulation of SL model in subsection-A and, required further modifications to take into account the impact of loading in subsection-B.

\section{A. Modeling of $S L$}

Various components of SL are, in fact,the additional eddy current losses $\left(P_{E d d y}\right)$ in response to the space and time harmonics. The expression for $P_{E d d y}$ for a sinusoidal excitation of flux-density magnitude $B_{m}$ and, frequency $f$ is given as:

$$
P_{E d d y}=k_{e} \omega^{2} B_{m}^{2}
$$

SL is the portion of the net $P_{E d d y}$ in a machine, generated by the space and time harmonics. These harmonics are generated from air-gap flux variation due to the slots, magnetic 
saturation and, leakage flux [8-11]. Net $P_{E d d y}$ produced by all the harmonics is:

$$
P_{E d d y}=k_{e} B_{m 1}^{2} \omega_{1}^{2}+\sum_{v=2}^{n} k_{e} B_{m v}^{2} \omega_{v}^{2}
$$

where, $B_{m v}$ and $\omega_{v}$ are the flux density magnitude and frequency of the $v$-th space harmonic,respectively. Denoting $k_{e} B_{m 1}^{2} \omega_{1}^{2}$ as $P_{E d d y 1}$ i.e., eddy current loss corresponding to the fundamental air-gap flux density $\left(B_{1}\right)$ and fundamental frequency $\left(\omega_{1}\right)$,

$$
P_{E d d y}=P_{E d d y_{1}}\left(1+\sum_{v=2}^{n}\left(\frac{B_{m v}}{B_{1}}\right)^{2}\left(\frac{\omega_{m v}}{\omega_{1}}\right)^{2}\right)
$$

The SL due to $v$-th harmonic is:

$$
S L_{V}=P_{E d d y 1}\left(\frac{B_{m v}}{B_{1}} \frac{\omega_{m v}}{\omega_{1}}\right)^{2}
$$

SL is hence,

$$
S L=P_{E d d y_{1}}\left(\sum_{v=2}^{n}\left(\frac{B_{m v}}{B_{1}}\right)^{2}\left(\frac{\omega_{m v}}{\omega_{1}}\right)^{2}\right)
$$

Two of major SL components in a non-skewed IM are surface eddy current losses and, tooth flux pulsation losses [4], [8], [13].

1) Surface eddy current losses: Modulation of the air-gap flux density due to slots results higher order space harmonics which creates additional eddy current losses on the surface of stator and rotor laminations. The impact of stator slot openings is prominent and hence, the surface losses produced by the rotor slots is negligible [13]. Harmonic numbers of the travelling flux-density waves of these harmonics are $v=N_{s} \pm p$ [13]. Frequency of the additional eddy currents induced on the rotor surface (corresponding to the $v=N_{s} \pm p$ ) is hence,

$$
f_{v}=s_{v} f_{1} \Rightarrow f_{v}=\left(1-\frac{N_{s} \pm p}{p}\right) f_{1}=\frac{N_{s}}{p} f_{1}
$$

The magnitudes of these harmonics are determined from the magnitudes of the variations in MMF due to slots as [15]

$$
\frac{B_{m v}}{B_{1}}=\frac{F_{m v} \lambda_{v}}{F_{1} \lambda_{1}}=\frac{F_{m v} \lambda_{s v}}{F_{1} \lambda_{1 s}}=\frac{L_{m s v}}{L_{m 1}}
$$

where, $F_{m v}, F_{1}$ are, respectively, the $v$-th harmonic and fundamental MMFs. $\lambda_{m}, \lambda_{1}$ are specific permeances of $v$ th harmonic and fundamental flux paths, respectively. $L_{m v}$ is magnetising inductance corresponding the $v$-th harmonic expressed as [9]

$$
L_{m v}=\frac{6 \mu_{o}\left(N_{s} K_{w}\right)^{2}}{\pi \cdot v^{2}}\left(\frac{L \cdot D}{p^{2} \cdot g \cdot K_{C} \cdot K_{S v}}\right)
$$

SL due to the eddy current losses corresponding to these harmonics (as per (5)) is:

$$
S L_{s u r f}=P_{E d d y_{1}}\left(\left(\frac{N_{s}}{p}\right)^{2} \sum_{v=2}^{n}\left(\frac{B_{m v}}{B_{1}}\right)^{2}\right)
$$

from $(7),(8)$

$$
S L_{\text {surf }}=P_{E d d y_{1}}\left(\left(\frac{N_{s}}{p}\right)^{2} \sum_{v=1}^{n}\left(\frac{L_{m s v}}{L_{m 1}}\right)^{2}\right)
$$

Using the expression for zig-zag leakage inductances (zig-zag leakage inductance is the sum of all the harmonic magnetising inductances other than fundamental [13]),

$$
L_{l z}=\sum_{v=2}^{n} L_{m v}
$$

From (10) and, (11),

$$
S L_{s u r f}=P_{E d d y_{1}}\left(\frac{N_{s}}{p}\right)^{2}\left(\frac{L_{l s z}}{L_{m} 1}\right)^{2}
$$

2) Flux pulsation losses: The variation in the main flux path permeance due to change in rotor position results in flux pulsation losses both in stator and rotor. The impact of rotor slot is very small in case closed or narrow-opening rotor slots. The frequency of eddy currents generated by the flux pulsation due to stator slot openings is $f_{v}=\frac{N_{s}}{p} f_{1}$ and, the same due to rotor slot openings is $f_{v}=\frac{N_{r}}{p} f_{1}$. The magnitudes of these harmonics are given as [4]

$$
B_{m t v}=K_{t v} B_{1}\left(\frac{t_{s}}{b_{s 0}}\right)
$$

where, neglecting the skewing,

$$
K_{t v}=1-\frac{L_{m v}+L_{l s z}}{L_{m v}+L_{l s z}+L_{S l o t}}=\frac{L_{S l o t}}{L_{m v}+L_{l s z}+L_{S l o t}}
$$

here, $L_{S l o t}$ is the slot leakage inductance. With $L_{m v} \gg$ $L_{\text {Slot }}+L_{l s z}$,

$$
B_{m t v}=\left(\frac{L_{S l o t}}{L_{m v}}\right)\left(\frac{t_{s}}{b_{s 0}}\right) B_{1}
$$

where, $t_{s}$ and, $b_{s 0}$, respectively represent the slot pitch and, slot opening. SL corresponding to these harmonics in stator is calculated as

$$
S L_{p u l s S}=P_{E d d y_{1}}\left(\left(\frac{N_{s}}{p}\right)^{2} \sum_{v=2}^{n}\left(\frac{B_{m t v}}{B_{1}}\right)^{2}\right)
$$

With using (13)-(16),

$$
S L_{p u l s S}=P_{E d d y_{1}}\left(\left(\frac{N_{s}}{p}\right)^{2}\left(\frac{L_{S l o t S}}{L_{m 1}} \frac{t_{s S}}{b_{s 0 S}}\right)^{2}\right)
$$

similarly, for rotor

$$
S L_{p u l s R}=P_{E d d y_{1}}\left(\left(\frac{N_{R}}{p}\right)^{2}\left(\frac{L_{S l o t R}}{L_{m 1}} \frac{t_{s R}}{b_{s 0 R}}\right)^{2}\right)
$$

With (12) and, (17)

$$
\begin{gathered}
S L=S L_{\text {surf }}+S L_{\text {puls } S}+S L_{\text {pulsR }} \\
\Rightarrow S L=P_{E d y_{1}}\left(\frac{N_{s}}{p}\right)^{2}\left(\begin{array}{l}
\left(\frac{L_{l s z}}{L_{m}}\right)^{2}+\left(k_{s} \frac{L_{\text {Slot } S}}{L_{m 1}}\right)^{2}+ \\
\left(k_{r} \frac{L_{S l o t R}}{L_{m 1}}\right)^{2}
\end{array}\right)
\end{gathered}
$$

where, $k_{s}=\frac{t_{s S}}{b_{s 0 S}}$ and, $k_{r}=\frac{N_{R} t_{s R}}{N_{S} b_{s 0 R}}$. Equation (20) is written in form of IM's output power with using the relations shown in $(21)-(24)$

$$
P_{\text {Out }}=3\left(\frac{\omega^{2} \lambda_{s}^{2}}{Z_{s}}\right) \cos \phi
$$


where, $\omega, \lambda_{s}, Z_{s}$ and, $\cos \phi$ denote supply frequency,stator flux linkage, input impedance and, power factor of a three phase IM. Rewriting (21) in terms of average air-gap flux density $(B)$ results:

$$
P_{\text {Out }}=3\left(\frac{\omega^{2}\left(T_{s}^{2} B^{2} A^{2}\right)}{Z_{s}}\right) \frac{R_{R}}{Z_{s}} \Rightarrow P_{\text {Out }}=3\left(\frac{T_{s}^{2} A^{2} R_{R}}{Z_{s}^{2}}\right) \omega^{2} B^{2}
$$

where, $T_{s}, A, R_{R}$ are, respectively, the stator number of turns/phase, area under a pole, and rotor resistance. The expression of eddy current loss is:

$$
P_{E d d y}=k_{e} \omega^{2} B^{2}
$$

Comparing (22) and (23),

$$
\Rightarrow P_{E d d y}=k^{2} P_{\text {Out }}
$$

From (20) and,(24),

$$
S L=k^{2}\left(\frac{1}{p}\right)^{2}\left(\begin{array}{l}
\left(\frac{L_{l s z}}{L_{m 1}}\right)^{2}+\left(k_{s} \frac{L_{\text {Slot } S}}{L_{m 1}}\right)^{2}+ \\
\left(k_{r} \frac{L_{\text {Slot } R}}{L_{m 1}}\right)^{2}
\end{array}\right) P_{\text {Out }}
$$

Since, in an IM $L_{l S l o t S} \gg L_{l z s}, L_{l S} \approx L_{l S l o t S}$ and, $L_{l R} \approx$ $L_{l \text { SlotR }}$, the expression (25), with denoting $L_{m 1}$ as $L_{m}$ can be written as

$$
S L=\left(\left(\frac{k k_{s}}{p^{2}} \frac{L_{l S}}{L_{m}}\right)^{2}+\left(\frac{k k_{r}}{p^{2}} \frac{L_{l R}}{L_{m}}\right)^{2}\right) P_{\text {Out }}
$$

3) Statistical evaluation: SL for 18 IMs of set- 1 is calculated as per (26) using the inductances $L_{m}, L_{l S}$ and, $L_{l R}$. The values of these inductances are given in annexure-3. However, high difference between the measured and calculated values with (26) (of the range $200 \%-300 \%$ ) suggests further improvement in (26). Hence, three another empirical representations of SL are explored using the same prime variables of (26) i.e., $P_{\text {Out }}, \frac{L_{l S}}{p L_{m}}, \frac{L_{l R}}{p L_{m}}$. The three empirical representations of SL are:

$$
\begin{aligned}
& S L=k\left(\left(\frac{1}{p} \frac{L_{l S}}{L_{m}}\right)^{m}+\left(\frac{1}{p} \frac{L_{l R}}{L_{m}}\right)^{n}\right) P_{\text {Out }} \\
& S L=k\left(\frac{1}{p} \frac{L_{l S}}{L_{m}}+\frac{1}{p} \frac{L_{l R}}{L_{m}}\right)^{m} P_{\text {Out }} \\
& S L=k\left(\frac{1}{p} \frac{L_{l S}}{L_{m}}\right)^{m}\left(\frac{1}{p} \frac{L_{l R}}{L_{m}}\right)^{n} P_{\text {Out }}
\end{aligned}
$$

where, $k$ is the proportional constant and, $m$ and $n$ are the exponents chosen to reflect the impact of rotor and stator slot geometries on the SL. These parameters are determined with the nonlinear least-squares regression. Values of the exponents and, calculated SL with these three models are given in annexure-3. The average errors with (26a),(26b) and (26c) are $42.42 \%, 25.89 \%$ and, $24.56 \%$, respectively. Standard deviation of errors (26a),(26b) and (26c) are $27.82 \%, 18.88 \%$ and, $15.44 \%$, respectively. The relative capability of these three models is further checked with relative-quality-estimates viz. Akaike Information Criterion (BIC) and Bayesian Information Criterion (BIC). The AIC and BIC weights are calculated for each model as per [16] using number of model parameters
( $n$ ) and, the residual sum of error-squares $\left(\sigma_{k}\right)$. Values of these scores are given in Table II. The model with least AIC and BIC score (or with zero values of $\triangle A I C$ and $\triangle B I C$ ) is considered the best estimator. The prediction capabilities $(C)$ of the three models are described as

$$
C_{26 c}>C_{26 b}>C_{26 a}
$$

TABLE II

QUALITY ESTIMATES OF SL MODELS

\begin{tabular}{ccccccc}
\hline \hline SL Model & $n$ & $\sigma_{k}$ & $A I C$ & $\Delta A I C$ & $B I C$ & $\Delta A I C$ \\
\hline 26a & 4.00 & 5.88 & -12.14 & 19.59 & -8.58 & 18.70 \\
26b & 5.00 & 3.43 & -19.83 & 11.91 & -15.38 & 11.91 \\
26c & 5.00 & 1.77 & -31.73 & 0 & -27.28 & 0 \\
\hline \hline
\end{tabular}

\section{B. Adapting SL model for loading condition}

The model development discussed in subsection-A uses a single value of measured SL and, single set of model parameters (i.e., $P_{O u t}, \frac{L_{l S}}{p L_{m}}, \frac{L_{l R}}{p L_{m}}$ ) for each of the $18 \mathrm{IMs}$ of set-1. This gives a linear trend of SL with $P_{\text {Out }}$. However, the variation of SL w.r.t. output power is not linear. Hence, the expression (26c) needs further modifications to account for the nonlinearity associated with the SL-P $P_{\text {Out }}$ relation. The following modifications in are feasible:

a. make $L_{l S}$ and $L_{l R}$ functions of stator and rotor currents, respectively.

b. make $L_{m}$ a function of stator current.

c. make the exponents $m$ and $n$ function of slip.

The $L_{l S}$ and $L_{l R}$ do not vary much with loading due to a large air in the path of leakage flux lines in comparison to the magnetising flux lines(till $I_{S} / I_{\text {Srated }}<2$ ) [13], [15]. Since the $L_{l S}$ and $L_{l R}$ do not show a significant variation w.r.t load current, they considered constant (as determined from no-load and block rotor test). On the other hand, the $L_{m}$ changes with the loading because variation in load results in a change of the magnetising voltage due to the change in stator resistive drop as:

$$
E_{m}=V_{S}-I R_{S}
$$

The magnetising flux-linkage corresponding to the is:

$$
\begin{gathered}
\psi_{m}=\int E_{m} d t=-\frac{1}{\omega_{e}} j E_{m} \\
L_{m}\left(\psi_{m}\right)=\frac{\psi_{m}}{I_{m}\left(\psi_{m}\right)}
\end{gathered}
$$

From the above expression, it is evident that $L_{m}$ is a function of flux-linkage $\psi_{m}$, and magnetising current $\left(I_{m}\right)$. Since both, $\psi_{m}$ and $I_{m}$ change due to loading, the $L_{m}$ also changes with loading. The variation of $L_{m}$ can is evaluated using the noload test at different voltages, and Fig.4(b) shows a typical $L_{m}$ variation. It is clear from Fig.4(b) that $L_{m}$ rises with an increase in load current. However, the $L_{m}$ variation (as shown in Fig.7(b)) does not take into account the impact of leakage fluxes as it uses no-load test data and the leakage flux is minimal at no-load conditions. 


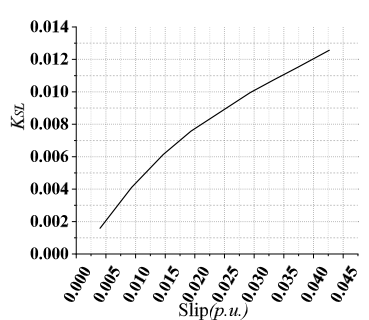

(a)

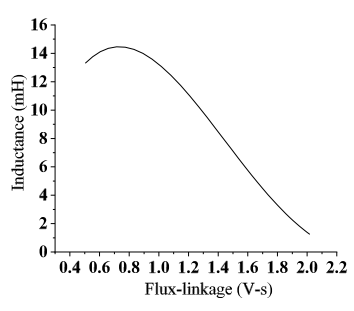

(b)
Fig. 4. Variations due to load-change: (a) $K_{S L}$ with slip for M-1, (b) $L_{m}$ with $\psi_{m}$.

The other factor that affects $L_{m}$ is tooth-bridge saturation. As the leakage fluxes increase with the load current, the tooth-bridge begins to saturate. Thereby, the permeance of the slots reduces leading to decrease in $L_{m}$. Since, both the above phenomena tend to have an opposing influence on $L_{m}$, the $L_{m}$ variation w.r.t loading is dependent on the motor's geometry. For example, a motor with a higher tooth width has a different $L_{m}$ variation compared to a motor with a narrower tooth. To incorporate the effect of $L_{m}$ variation on SL (i.e. flux dependent variation), the relation (26c) is modified for load-dependent $L_{m}$ as:

$$
K_{S L}=\frac{S L}{P_{\text {Out }}}=\left(\frac{1}{p} \frac{L_{l s}}{L_{m}\left(I_{m}\right)}\right)^{m}\left(\frac{1}{p} \frac{L_{l r}}{L_{m}\left(I_{m}\right)}\right)^{n}
$$

The third option is to make the exponents $m$ and $n$ function of slip. Hence, based on the above discussion, the modified version of (27) is:

$$
K_{S L}=\left(\frac{1}{p} \frac{L_{l S}}{L_{m}\left(I_{m}\right)}\right)^{m(s)}\left(\frac{1}{p} \frac{L_{l R}}{L_{m}\left(I_{m}\right)}\right)^{n(s)}
$$

To keep the number of variables small, exponent $n(s)$ is taken as a constant multiplier of $m(s)$ with zero offset, i.e. $n(s)=k \cdot m(s)$. Using this, (28) becomes:

$$
K_{S L}=\left(\frac{1}{p} \frac{L_{l S}}{L_{m}\left(I_{m}\right)}\right)^{m(s)}\left(\frac{1}{p} \frac{L_{l R}}{L_{m}\left(I_{m}\right)}\right)^{k \cdot m(s)}
$$

Determination of the exponents $m(s)$ and $n(s)$ uses measured

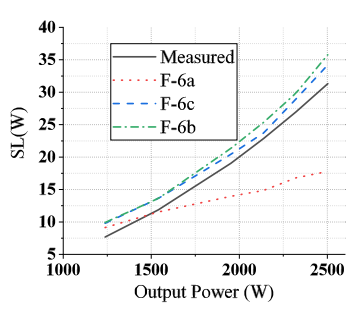

(a)

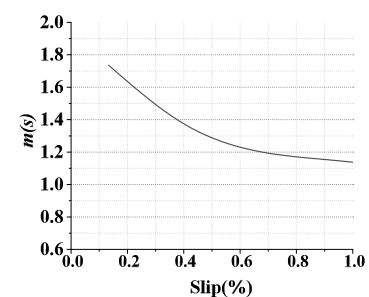

(b)
Fig. 5. (a) SL variation: Measured and estimated with the three variants of SL model for M-1, (b) Variation of the exponent $m(s)$ corresponding to (30)

values of SL fractions in total output power i.e., $S L / P_{\text {Out }}$ at different loadings of the motor. This fraction is the measure of $K_{S L}$ by its definition. III-B shows the $K_{S L}$ variation for the motor M-1. The relation between these $K_{S L}$ values and the $m(s)$ is given as:

$$
\frac{1}{m(s)} \log \left(K_{S L}\right)=\log (A)+k \log (B)
$$

where,

$$
A=\frac{1}{p}\left(\frac{L_{l S}}{L_{m}}\right) \text { and, } B=\frac{1}{p}\left(\frac{L_{l R}}{L_{m}}\right) .
$$

The right term in (30) is solely a geometry and material dependant term and can be evaluated during the design or the testing. However, neglecting the saturation makes this term a constant and as a result, the variation of $m(s)$ w.r.t. loading (or the $P_{O u t}$ or the measured $K_{S L}$ ) will always lead to a constant:

$$
\frac{1}{m(s)} \log \left(K_{S L}\right)
$$

The $m(s)$ variation meeting this constraint with correlation $R^{2}=0.99$ is deduced as:

$$
\begin{gathered}
m(s)=(1-s)^{\sqrt{2} \pi} \\
k=\frac{1}{2 \sqrt{2}}
\end{gathered}
$$

Fig.5(b) shows the trend of $m(s)$ correspond to the constraint (30) for the motor M-1.

\section{VALIDATION AND DESIGN IMPLiCATION OF THE MODIFIED SL MODEL}

This section presents the validation of the proposed load dependant SL model wherein, the SL measurements of eight $2.5 \mathrm{~kW}$ prototype IMs are used. In further step, a $75 \mathrm{~kW}$ IM is re-designed in congruence with the proposed model's notion to verify the model's capability. Subsection-A and B detail the analysis for the eight IMs and, the $75 \mathrm{~kW}$ IM, respectively. Stator tooth flux density variation is used to correlate the model's predictions.

\section{A. Validation with Motors of Same Power}

Three variants of (29), inferred from the discussion in subsection-B of the previous section, are used to validate the proposed method and, these are:

a. model with variable $L_{m}$ and constant exponents ( $m$ and $n$ ). (i.e., expression (29) with load-dependent $L_{m}$ ): F-6a;

b. model with constant $L_{m}$. (i.e., expression (29) with rated $L_{m}$ values): F-6b

c. model with load-dependent $L_{m}$ : F-6c.

SL estimates with the three variants of the model for the eight IMs are shown in Table III.

The careful investigation of these tables and, Fig.5, shows that:

- the model F-6a always underestimates the SL w.r.t the measurement. Also, the trend of SL variation with F-6a, as shown in Fig.5(a), is different from the measurement. The mismatch in the trend asserts the need for loaddependent exponents $m$ and $n$.

- model F-6b and F-6c show SL trend similar to the measurement. However, the variant F-6b always overestimates SL. 
TABLE III

Measured and Estimated SL Values with Modified SL Model VARIANTS

\begin{tabular}{|c|c|c|c|c|c|}
\hline \multirow{2}{*}{ IM } & \multirow{2}{*}{$\begin{array}{l}P_{\text {Out }} \\
(\mathrm{kW})\end{array}$} & \multirow{2}{*}{$\begin{array}{c}\text { SL } \\
\text { (Measured) }\end{array}$} & \multicolumn{3}{|c|}{ Estimated SL with: } \\
\hline & & & F-6a & F-6b & F-6c \\
\hline \multirow{4}{*}{ M-1 } & 1.95 & 19.05 & 13.95 & 21.34 & 20.43 \\
\hline & 2.14 & 22.86 & 14.87 & 25.37 & 23.72 \\
\hline & 2.32 & 26.98 & 16.81 & 29.89 & 28.99 \\
\hline & 2.5 & 31.32 & 17.79 & 35.80 & 34.22 \\
\hline \multirow{4}{*}{ M-2 } & 1.95 & 15.26 & 12.05 & 20.73 & 17.61 \\
\hline & 2.14 & 18.34 & 13.03 & 24.35 & 20.47 \\
\hline & 2.32 & 21.62 & 14.72 & 29.07 & 25.42 \\
\hline & 2.5 & 25.09 & 15.49 & 34.84 & 29.94 \\
\hline \multirow{4}{*}{ M-3 } & 1.95 & 15.24 & 12.05 & 19.43 & 17.60 \\
\hline & 2.14 & 18.29 & 13.03 & 23.14 & 20.93 \\
\hline & 2.32 & 21.59 & 14.72 & 27.67 & 26.08 \\
\hline & 2.5 & 25.05 & 15.49 & 33.21 & 29.83 \\
\hline \multirow{4}{*}{ M-4 } & 1.95 & 18.31 & 13.14 & 20.14 & 19.08 \\
\hline & 2.14 & 21.92 & 14.59 & 24.61 & 23.66 \\
\hline & 2.32 & 25.83 & 16.60 & 29.37 & 29.45 \\
\hline & 2.5 & 29.14 & 16.56 & 35.65 & 33.48 \\
\hline \multirow{4}{*}{ M-5 } & 1.95 & 28.58 & 13.86 & 30.72 & 29.27 \\
\hline & 2.14 & 34.29 & 15.56 & 39.28 & 38.24 \\
\hline & 2.32 & 40.48 & 17.86 & 46.98 & 47.84 \\
\hline & 2.5 & 46.98 & 17.41 & 64.34 & 60.70 \\
\hline \multirow{4}{*}{ M-6 } & 1.95 & 25.27 & 11.92 & 29.00 & 24.71 \\
\hline & 2.14 & 30.32 & 13.56 & 36.60 & 32.36 \\
\hline & 2.32 & 35.79 & 15.57 & 45.65 & 42.36 \\
\hline & 2.5 & 41.54 & 15.09 & 59.72 & 51.30 \\
\hline \multirow{4}{*}{ M-7 } & 1.95 & 23.04 & 12.11 & 24.89 & 22.47 \\
\hline & 2.14 & 27.65 & 13.92 & 35.44 & 33.49 \\
\hline & 2.32 & 32.64 & 16.05 & 44.14 & 43.82 \\
\hline & 2.5 & 37.88 & 15.04 & 56.65 & 50.57 \\
\hline \multirow{4}{*}{ M-8 } & 1.95 & 26.46 & 13.00 & 26.78 & 25.23 \\
\hline & 2.14 & 31.75 & 15.22 & 30.52 & 30.47 \\
\hline & 2.32 & 37.48 & 17.59 & 45.07 & 47.28 \\
\hline & 2.5 & 43.50 & 16.17 & 56.93 & 52.79 \\
\hline
\end{tabular}

- the SL estimates with F-6c have $8.13 \%$ (22.57\%-14.44\%, as shown in Table IV) lesser average error in comparison to F-6b. This suggests that the variation of $L_{m}$ if taken into account gives SL values closer to the measurement in variable slip models.

- the motors M-1 to M-8 have the same specifications (as shown in annexure-2) but different slot geometries. Due to the difference in slot geometry, they have different levels of distortions in stator-tooth flux densities (as shown in Fig. 6) measured SL values. The variants F$6 \mathrm{~b}$ and F- $6 \mathrm{c}$ also give different values of SL for the eight motors.

- the slip-dependent exponents make the model a function of the rotor-bar material. The motors M-1 and M-6 have same geometry with $\mathrm{M}-1$ having a copper $(\mathrm{Cu})$ rotor and M-6 an aluminium (Al) rotor. Since the $\mathrm{Al}$ rotor runs at higher slip than $\mathrm{Cu}$ rotor for the same Pout, its exponents in (3) will be lesser than $\mathrm{Cu}$ motor. Hence, the SL of $\mathrm{Al}$ motor will be higher than the $\mathrm{Cu}$ motor (as shown in column-6: row-17-20 and row-2-5).

From the above discussion, it is clear that both the modified variants F-6b and F-6c (the models with variable exponents) predict the SL trend with a small relative difference. The variant F-6c needs multiple tests to encase the $L_{m}$ variation while F-6b uses only one $L_{m}$ value. Hence, F-6b is a convenient
TABLE IV

Percentage ERrors In Calculated SL Values

\begin{tabular}{|c|c|c|c|c|c|}
\hline \multirow{2}{*}{ IM } & \multirow{2}{*}{$\begin{array}{c}P_{\text {Out }} \\
(\mathrm{kW})\end{array}$} & \multirow{2}{*}{$\begin{array}{c}\text { SL } \\
\text { (Measured) }\end{array}$} & \multicolumn{3}{|c|}{ Percentage error with: } \\
\hline & & & F-6a & F-6b & F-6c \\
\hline \multirow{4}{*}{ M-1 } & 1.95 & 19.05 & 26.78 & 12.00 & 7.25 \\
\hline & 2.14 & 22.86 & 34.95 & 10.99 & 3.78 \\
\hline & 2.32 & 26.98 & 37.69 & 10.77 & 7.43 \\
\hline & 2.5 & 31.32 & 43.19 & 14.30 & 9.26 \\
\hline \multirow{4}{*}{ M-2 } & 1.95 & 15.26 & 21.02 & 35.87 & 15.40 \\
\hline & 2.14 & 18.34 & 28.94 & 32.76 & 11.63 \\
\hline & 2.32 & 21.62 & 31.91 & 34.46 & 17.56 \\
\hline & 2.5 & 25.09 & 38.26 & 38.88 & 19.35 \\
\hline \multirow{4}{*}{ M-3 } & 1.95 & 15.24 & 20.92 & 27.47 & 15.49 \\
\hline & 2.14 & 18.29 & 28.75 & 26.50 & 14.44 \\
\hline & 2.32 & 21.59 & 31.82 & 28.17 & 20.82 \\
\hline & 2.5 & 25.05 & 38.17 & 32.56 & 19.10 \\
\hline \multirow{4}{*}{ M-4 } & 1.95 & 18.31 & 28.23 & 10.00 & 4.19 \\
\hline & 2.14 & 21.92 & 33.43 & 12.26 & 7.93 \\
\hline & 2.32 & 25.83 & 35.75 & 13.72 & 14.01 \\
\hline & 2.5 & 29.14 & 43.17 & 22.33 & 14.90 \\
\hline \multirow{4}{*}{ M-5 } & 1.95 & 28.58 & 51.51 & 7.52 & 2.42 \\
\hline & 2.14 & 34.29 & 54.63 & 14.56 & 11.54 \\
\hline & 2.32 & 33.45 & 55.86 & 16.07 & 18.19 \\
\hline & 2.5 & 38.82 & 62.95 & 36.96 & 29.22 \\
\hline \multirow{4}{*}{ M-6 } & 1.95 & 25.27 & 52.83 & 14.77 & 2.21 \\
\hline & 2.14 & 30.32 & 55.26 & 20.72 & 6.73 \\
\hline & 2.32 & 35.79 & 56.49 & 27.54 & 18.34 \\
\hline & 2.5 & 41.54 & 63.68 & 43.76 & 23.49 \\
\hline \multirow{4}{*}{ M-7 } & 1.95 & 23.04 & 47.43 & 8.02 & 2.49 \\
\hline & 2.14 & 27.65 & 49.64 & 28.16 & 21.11 \\
\hline & 2.32 & 32.64 & 50.82 & 35.22 & 34.23 \\
\hline & 2.5 & 37.88 & 60.30 & 49.53 & 33.49 \\
\hline \multirow{4}{*}{ M-8 } & 1.95 & 26.46 & 50.88 & 1.21 & 4.66 \\
\hline & 2.14 & 31.75 & 52.06 & 3.86 & 4.04 \\
\hline & 2.32 & 37.48 & 53.05 & 20.27 & 26.15 \\
\hline & 2.5 & 43.50 & 62.81 & 30.88 & 21.36 \\
\hline \multicolumn{3}{|c|}{ Average error: } & 43.85 & 22.57 & 14.44 \\
\hline \multicolumn{3}{|c|}{ Standard deviation: } & 12.76 & 12.37 & 9.01 \\
\hline
\end{tabular}

choice.

\section{B. Validation with Design of $75 \mathrm{~kW}$ IM}

This section explains the use of the proposed model in designing an IM for lower SL. For this, an existing 4-pole, $400 \mathrm{~V}, 75 \mathrm{~kW}$ IM's stator, and rotor slot are redesigned to achieve a lower SL. It is noteworthy to mention here that, the original motor already possesses un-skewed slots and 5/6 short-pitched stator windings- the design attributes of lower SLThe design method used here to lower the SL uses these two prime variables. Since the winding scheme and geometries other than slot geometries are unchanged during modifications, the objective to reduce $L_{l S} / L_{m}$ and $L_{l R} / L_{m}$ in (6) can be summarised as reduction of term:

$$
F_{o b j}=K_{C} K_{S}\left(\lambda_{\lg }+\lambda_{l S S}\right)
$$

Slot areas are kept same while applying the (34). The changes in the initial IM are:

- variation airgap conductance harmonics $\left(a_{n}\right)$ with the slot opening is recorded and the $B_{S 0}$ of stator is set to the minimum value constrained by the winding placement. In response, the carter's coefficient and $a_{n}$ are reduced and consequently, the $L_{m}$ is improved. (Fig.7 shows the magnitudes of $a_{n}$ up to 4 th order for both the IMs.) 

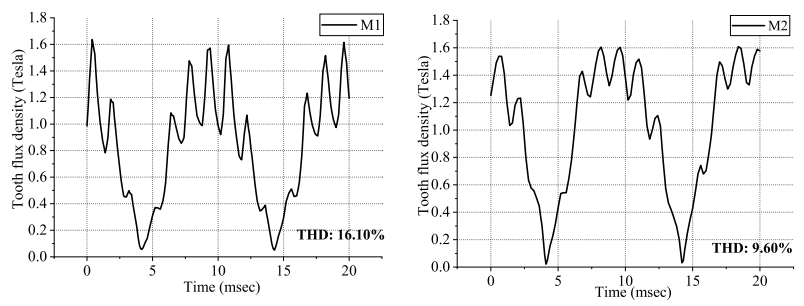

(a) Tooth flux density $\left(B_{t}\right)$ in M1 (b) Tooth flux density $\left(B_{t}\right)$ in M2
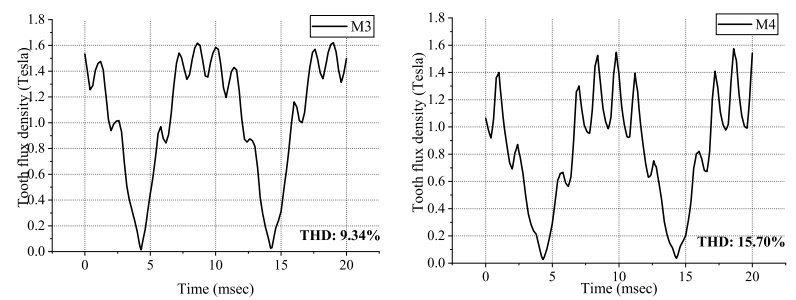

(c) Tooth flux density $\left(B_{t}\right)$ in M3 (d) Tooth flux density $\left(B_{t}\right)$ in M4

Fig. 6. Stator tooth flux density: for four $2.5 \mathrm{~kW}$ IMs of copper cage rotor

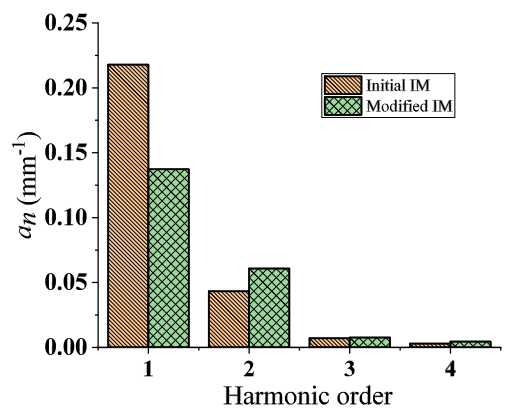

Fig. 7. Harmonic spectrum of airgap conductance for the initial and modified $75 \mathrm{~kW}$ IM.

- $B_{S 1}$ is made zero and the tooth width is increased by $1 \mathrm{~mm}$ to reduce the $a_{n}$.

- $H_{S 2}$ is increased slightly to compensate the decrease in $\lambda_{l S S}$ due to lower $B_{S 0}$ and $H_{S 1}$ (to limit the blocked rotor current).

- in rotor slot,all the sharp edges are replaced with arcs to avoid the leakage flux concentration. $B_{S 0}$ and $B_{S 0}$ are increased and to keep the net rotor leakage inductance as it is,slot height is slightly increased.

Table $\mathrm{V}$ shows the initial and modified slot shapes of the $75 \mathrm{~kW}$ IMs.

1) Experimental determination of SL: Both the modified and original $75 \mathrm{~kW}$ motors are tested for variable SL by changing the load at $400 \mathrm{~V}, 50 \mathrm{~Hz}$ sinusoidal power supply. The SL measurement is done as per the procedure in IEC600342-1, section 8.2.2.5. Table VI and VII show the test results.

2) Verification of the proposed modified model F-6b: The calculated values of SL using the proposed modified SL model are shown in Table VIII and Table IX for the original and modified $75 \mathrm{~kW}$ IM, respectively.

Careful examination of these values reveals that:

- the motor designed with modified geometry has $4.5 \%$ smaller SL in comparison to the original IM.

- despite both IMs having the same power ratings, the
TABLE V

SLOT Shapes OF THE INITIAL AND MOdified 75KW IM

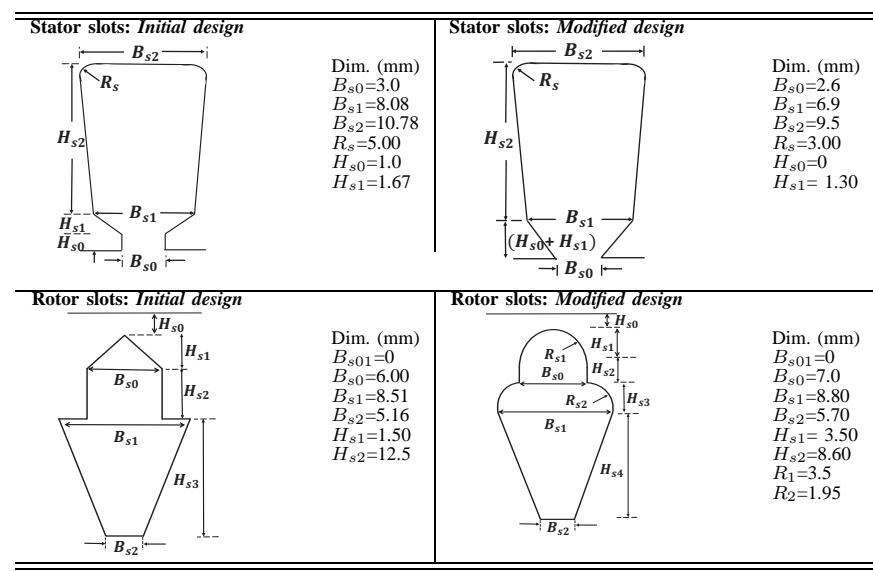

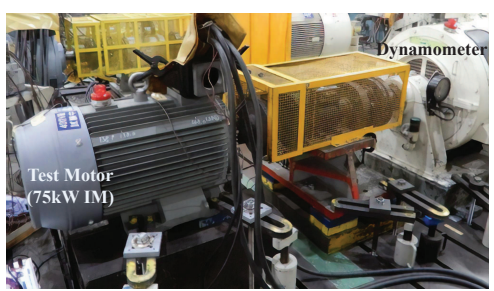

Fig. 8. Test bench with the $75 \mathrm{~kW}$ IM

number of poles, winding schemes and volumes, the proposed model is capable enough to distinguish them for SL.

- though the model captures the SL trend in line with measurement, the error in SL prediction is high at light load and overload conditions.

- the results with the modified model show correlation coefficients of 0.99 with the corresponding measured SL values for both the $75 \mathrm{~kW}$ IMs.

\section{CONCLUSION}

This work presents a stray loss model for a grid-connected IM for variable loading. The development of the model uses measurement data of 8 different IMs. The model is developed

TABLE VI

TEST RESULTS FOR SL OF ORIGINAL IM

\begin{tabular}{ccccccc}
\hline \hline $\begin{array}{c}\text { Sr. } \\
\text { No. }\end{array}$ & $\begin{array}{c}\text { Load } \\
(\%)\end{array}$ & $\begin{array}{c}\text { Supply } \\
\text { Voltage (V) }\end{array}$ & $\begin{array}{c}\text { Current } \\
(\mathrm{A})\end{array}$ & $\begin{array}{c}\text { Slip } \\
(\%)\end{array}$ & $\begin{array}{c}\text { Power } \\
\text { factor }\end{array}$ & $\begin{array}{c}\text { SL } \\
(\mathrm{W})\end{array}$ \\
\hline 1 & 50 & 400 & 77.1 & 0.51 & 74.2 & 106 \\
2 & 75 & 400 & 102.6 & 0.79 & 83.3 & 237 \\
3 & 100 & 400 & 131 & 1.08 & 87.1 & 425 \\
4 & 125 & 400 & 161.5 & 1.39 & 88.8 & 659 \\
5 & 150 & 400 & 193.6 & 1.7 & 89.4 & 956 \\
\hline \hline
\end{tabular}

TABLE VII

TEST RESUlts FOR SL OF MODIFIEd IM

\begin{tabular}{ccccccc}
\hline \hline $\begin{array}{c}\text { Sr. } \\
\text { No. }\end{array}$ & $\begin{array}{c}\text { Load } \\
(\%)\end{array}$ & $\begin{array}{c}\text { Supply } \\
\text { Voltage (V) }\end{array}$ & $\begin{array}{c}\text { Current } \\
(\mathrm{A})\end{array}$ & $\begin{array}{c}\text { Slip } \\
(\%)\end{array}$ & $\begin{array}{c}\text { Power } \\
\text { factor }\end{array}$ & $\begin{array}{c}\text { SL } \\
(\mathrm{W})\end{array}$ \\
\hline 1 & 50 & 400 & 76.8 & 0.55 & 74.1 & 104 \\
2 & 75 & 400 & 102.7 & 0.84 & 82.9 & 229 \\
3 & 100 & 400 & 131.6 & 1.17 & 86.6 & 406 \\
4 & 125 & 400 & 162.5 & 1.48 & 88.2 & 641 \\
5 & 150 & 400 & 195.6 & 1.83 & 88.7 & 915 \\
\hline \hline
\end{tabular}


TABLE VIII

Calculated and Measured SL:Original 75KW IM

\begin{tabular}{cccccccc}
\hline \hline $\begin{array}{c}\text { Sr. } \\
\text { No. }\end{array}$ & $\begin{array}{c}\text { Pout } \\
(\mathrm{kW})\end{array}$ & $\begin{array}{c}\text { Slip } \\
(\mathrm{pu})\end{array}$ & $m(s)$ & $n(s)$ & $\begin{array}{c}\text { SL } \\
(\mathrm{cal}-\mathrm{W})\end{array}$ & $\begin{array}{c}\text { SL } \\
(\mathrm{meas}-\mathrm{W})\end{array}$ & $\begin{array}{c}\% \\
\text { error }\end{array}$ \\
\hline 1 & 37.5 & 0.01 & 0.98 & 0.35 & 149.16 & 106 & 40.72 \\
2 & 56.25 & 0.01 & 0.96 & 0.34 & 240.25 & 237 & 1.37 \\
3 & 75 & 0.01 & 0.95 & 0.34 & 347.07 & 425 & 18.34 \\
4 & 93.75 & 0.01 & 0.94 & 0.33 & 467.38 & 659 & 29.08 \\
5 & 112.5 & 0.02 & 0.92 & 0.33 & 609.46 & 956 & 36.25 \\
\hline \multicolumn{7}{l}{ Average error: } \\
\multicolumn{7}{l}{ Ctandard deviation of error: } \\
\hline \multicolumn{7}{l}{ Correlation coefficient: } \\
\hline \hline
\end{tabular}

TABLE IX

Calculated and Measured SL:Modified 75KW IM

\begin{tabular}{cccccccc}
\hline \hline $\begin{array}{c}\text { Sr. } \\
\text { No. }\end{array}$ & $\begin{array}{c}P_{\text {Out }} \\
(\mathrm{kW})\end{array}$ & $\begin{array}{c}\text { Slip } \\
(\mathrm{pu})\end{array}$ & $m(s)$ & $n(s)$ & $\begin{array}{c}\text { SL } \\
(\mathrm{cal}-\mathrm{W})\end{array}$ & $\begin{array}{c}\text { SL } \\
(\mathrm{meas}-\mathrm{W})\end{array}$ & $\begin{array}{c}\% \\
\text { error }\end{array}$ \\
\hline 1 & 37.5 & 0.01 & 0.98 & 0.35 & 125.88 & 104 & 21.04 \\
2 & 56.25 & 0.01 & 0.97 & 0.34 & 202.68 & 229 & 11.49 \\
3 & 75 & 0.01 & 0.95 & 0.34 & 290.61 & 406 & 28.42 \\
4 & 93.75 & 0.01 & 0.94 & 0.34 & 392.28 & 641 & 38.8 \\
5 & 112.5 & 0.02 & 0.93 & 0.33 & 507.92 & 915 & 44.49 \\
\hline \multicolumn{7}{l}{ Average error: } \\
\multicolumn{7}{l}{ Standard deviation of error: } \\
\multicolumn{7}{l}{ Correlation coefficient: }
\end{tabular}

by testing the eight numbers of different small power IMs of same power ratings at different loads. The design applicability of the model is verified successfully by re-designing a medium power $75 \mathrm{~kW}$ IM for SL minimisation. The results comparing the measured and estimated SL with the proposed model lay the following conclusions:

- The model is capable of determining the SL for both the objectives: SL at design stage and SL for a motor in operation.

- SL is not the same, even when the motors have the same output specifications. The proposed model is capable of segregating the IMs of the same specifications and different geometries for their SL values, which helps in designing an IM of smaller SL.

- SL in an IM is load dependent loss; the proposed model expresses this variation in line with the measurements. This is experimentally verified for both the small and medium power IMs here.

- The modified model also incorporates the impact of rotor bar material on SL in terms of change in the exponents formulated.

\section{REFERENCES}

[1] “Annual report 2017," IEA Technology Collaboration Programme on Energy Efficient End-Use Equipment (4E), 2017.

[2] J. Cheaytani, A. Benabou, A. Tounzi, and M. Dessoude, "Stray load losses analysis of cage induction motor using 3-d finite-element method with external circuit coupling," IEEE Transactions on Magnetics, vol. 53, no. 6, pp. 1-4, 2017.

[3] N. Glew, "Stray load losses in induction motors: a challenge to academia," Power Engineering Journal, vol. 12, no. 1, pp. 27-32, Feb 1998.

[4] S. A. N. Donald W. Novotny, "High frequency losses in induction motors," NASA Lewis Research Center, June 1991.

[5] "Rotating electrical machines - part 2-1:standard methods for determining losses and efficiency from tests (excluding machines for traction vehicles)," IEC Std 60034-2-1, Sep. 2007.

[6] A. I. de Almeida, F. J. T. E. Ferreira, J. F. Busch, and P. Angers, "Comparative analysis of ieee 112-b and iec 34-2 efficiency testing standards using stray load losses in low-voltage three-phase, cage induction motors," IEEE Trans. Ind. Appl., vol. 38, no. 2, pp. 608-614, March 2002.

[7] M. Aoulkadi and A. Binder, "Comparison of different evaluation methods to determine stray load losses in induction machines with eh-star method," IEEE Trans. Ind. Appl., vol. 44, no. 6, pp. 1675-1682, Nov 2008.

[8] J. Wang, M. Cheng, H. Wen, M. Tong, G. Zhang, Y. Hu, and B. Chen, "Stray load loss calculation for induction motor by combination of general airgap field modulation theory and $2 \mathrm{~d}$ fea," IEEE Transactions on Energy Conversion, pp. 1-1, 2021.

[9] P. L. Alger, Induction Machines. Taylor \& Francis, 1995. [Online]. Available: https://books.google.co.in/books?id=pyBJPwAACAAJ

[10] P. Pillay, M. Al-Badri, P. Angers, and C. Desai, "A new stray-load loss formula for small and medium-sized induction motors," IEEE Trans. Energy Convers., vol. 31, no. 3, pp. 1221-1227, Sep. 2016.

[11] M. Basic and D. Vukadinovic, "Small size induction machine equivalent circuit including variable stray load and iron losses," Journal of Electrical Engineering and Technology, vol. 13, no. 2, pp. 1603-1612, 2018.

[12] S. Rahman, "Energy, economics and environmental analysis for chillers in office buildings," Energy Education Science and Technology Part aEnergy Science and Research, vol. 25, pp. 1-16, 042010.

[13] I. Boldea and S. A. Nasar, "Induction machines design handbook," vol. 31, no. 3, 2010.

[14] "Ieee standard test procedure for polyphase induction motors and generators," IEEE Std 112-2004 (Revision of IEEE Std 112-1996), pp. 1-83, Nov 2004.

[15] B.Heller and V.Hamata, "Harmonic field effects in induction machines," 1977.

[16] R. E. Kass and A. E. Raftery, "Bayes factors," Journal of the American Statistical Association, vol. 90, no. 430, pp. 773-795, 1995. 